\title{
On the Controllability of a Slowly Rotating Timoshenko Beam
}

\author{
W. Krabs and G. M. Sklyar
}

\begin{abstract}
We consider a slowly rotating Timoshenko beam in a horizontal plane whose movement is controlled by the angular acceleration of the disk of a driving motor into which the beam is clamped. The problem to be solved is to transfer the beam from a position of rest into a position of rest under a given angle within a given time. We show that this problem is solvable, if the time of rotation prescribed is large enough.
\end{abstract}

Keywords: Controllability, Timoshenko Beam

AMS subject classification: 93 B 05

\section{Introduction: the model and the problem of controllability}

The control of rotating beams has been the subject of several investigations during the last two decades. The majority of publications concentrated on the Euler beam model. So Sakawa and co-authors in [9] derived a nonlinear model of a rotating Euler beam in a horizontal plane and investigated the problem of controllability computationally. This model was picked up by Krabs in [5] and treated by theoretical methods. In particular, an iteration method for the solution of the problem of controllability was developed and investigated with respect to convergence. In [6] it was shown, however, that exact controllability is not possible, but that the solution method developed in [5] leads to approximative solutions of sufficient accuracy for practical purposes already after two steps.

In $[1,2]$ a linear model for a rotating Euler beam in a horizontal plane was derived. This model was investigated by Leugering in [7, 8] and by Krabs in [4].

Recently Xiao-Jin Xiong in his PhD thesis [10] derived a nonlinear model for a rotating Timoshenko beam in a horizontal plane, proved the well-posedness of its model equations, and gave a numerical method for solving the problem of controllability together with numerical examples. He also linearized the problem for the case of a slowly moving beam where in a dimension-free formulation the deflection $w(x, t)$ of the center line of the beam at the location $x \in[0,1]$ and time $t \geq 0$ and the rotation angle $\xi(x, t)$

W. Krabs: Techn. Univ., FB Mathematik, Schloßgartenstr. 7, D - 64289 Darmstadt

G. M. Sklyar: State Univ., Dept. Math. Anal., Svoboda sqr. 4, 310077 Kharkov, Ukraine and Universitet Szczecinski, Institut Matematyki, ul. Wielkopolska 15, Szczecin, Poland .

This research was supported by NATO Linkage Grant No. CRG-LG 972980

ISSN 0232-2064 / \$2.50 (C) Heldermann Verlag Berlin 
of the cross section area at $x$ and $t$ are governed by the two differential equations

$$
\left.\begin{array}{rl}
\ddot{w}(x, t)-w^{\prime \prime}(x, t)-\xi^{\prime}(x, t) & =-\ddot{\theta}(t)(r+x) \\
\ddot{\xi}(x, t)-\xi^{\prime \prime}(x, t)+\xi(x, t)+w^{\prime}(x, t) & =\ddot{\theta}(t)
\end{array}\right\} \quad(x \in(0,1), t>0)
$$

where $\dot{w}=w_{t}, \dot{\xi}=\xi_{t}$ and $w^{\prime}=w_{x}, \xi^{\prime}=\xi_{x}, \theta$ is the rotation angle of the motor disk, $\dot{\theta}=\frac{d \theta}{d t}$, and $r$ is the radius of the disk. In addition, we have boundary conditions of the form

$$
\left.\begin{array}{rl}
w(0, t)=\xi(0, t) & =0 \\
w^{\prime}(1, t)+\xi(1, t) & =0 \\
\xi^{\prime}(1, t) & =0
\end{array}\right\} \quad(t \geq 0)
$$

We assume the beam to be in the position of rest at $t=0$ which leads to the initial conditions

$$
\left.\begin{array}{cc}
w(x, 0)=\dot{w}(x, 0)=\xi(x, 0)=\dot{\xi}(x, 0)=0 & (x \in[0,1]) \\
\theta(0)=\dot{\theta}(0)=0 . &
\end{array}\right\}
$$

The motion of the beam is controlled by the acceleration $\ddot{\theta}(t)$ of the rotation angle of the motor disk.

In this paper we consider the following

Problem of Controllability: Given $T>0$ and $\theta_{T} \in \mathbb{R}$, find

$$
\theta \in H_{0}^{2}(0, T)=\left\{\theta \in H^{2}(0, T) \mid \theta(0)=\dot{\theta}(0)=0\right\}
$$

such that

$$
\left.\begin{array}{l}
\theta(T)=\theta_{T} \\
\dot{\theta}(T)=0
\end{array}\right\}
$$

and the "solution" $(w, \xi)$ of problem (1.1) - (1.3) satisfies the end conditions

$$
w(x, T)=\dot{w}(x, T)=\xi(x, T)=\dot{\xi}(x, T)=0 \quad(x \in[0,1]) .
$$

In addition to $\ddot{\theta}, \mathrm{Xiao-Jin}$ Xiong also considers boundary control of the form

$$
w^{\prime}(1, t)+\xi(1, t)=u(t) \quad \text { or } \quad \xi^{\prime}(1, t)=u(t) \quad \text { for } t \geq 0
$$

where $u=u(t)$ is a second control function taken from a suitable function space. Instead of (1.5) he considers end conditions of the form

$$
\left.\begin{array}{rl}
w(x, T)=w_{T}(x), & \dot{w}(x, T)=\dot{w}_{T}(x) \\
\xi(x, T)=\xi_{T}(x), \quad \dot{\xi}(x, T)=\dot{\xi}_{T}(x)
\end{array}\right\} \quad(x \in[0, T])
$$

where $w_{T}, \dot{w}_{T}$ and $\xi_{T}, \dot{\xi}_{T}$ are chosen in suitable function spaces. He shows that controllability is possible, if $T>0$ is large enough. 


\section{On the solution of the model equations}

Let $H=L^{2}\left((0,1), \mathbb{R}^{2}\right)$. Then we define a linear operator $A: D(A) \rightarrow H$ by

$$
A\left(\begin{array}{c}
y \\
z
\end{array}\right)=\left(\begin{array}{c}
-y^{\prime \prime}-z^{\prime} \\
y^{\prime}-z^{\prime \prime}+z
\end{array}\right)
$$

for $\left(\begin{array}{l}y \\ z\end{array}\right) \in D(A)$ where

$$
D(A)=\left\{\left(\begin{array}{l}
y \\
z
\end{array}\right) \in H^{2}\left((0,1), \mathbb{R}^{2}\right) \mid \begin{array}{l}
y(0)=z(0)=0 \\
y^{\prime}(1)+z(1)=0, z^{\prime}(1)=0
\end{array}\right\} .
$$

With this operator (1.1) can be rewritten in the form

$$
\left(\begin{array}{c}
\ddot{w}(\cdot, t) \\
\ddot{\xi}(\cdot, t)
\end{array}\right)+A\left(\begin{array}{c}
w(\cdot, t) \\
\xi(\cdot, t)
\end{array}\right)=\left(\begin{array}{c}
f_{1}(\cdot, t) \\
f_{2}(\cdot, t)
\end{array}\right) \quad(t>0)
$$

where

$$
\left.\begin{array}{l}
f_{1}(x, t)=-\ddot{\theta}(t)(r+x) \\
f_{2}(x, t)=\ddot{\theta}(t)
\end{array}\right\} \quad(x \in(0,1), t>0) .
$$

Let $\left(\begin{array}{l}y \\ z\end{array}\right) \in D(A)$ be given. Then it follows that

$$
\left\langle\left(\begin{array}{l}
y \\
z
\end{array}\right), A\left(\begin{array}{l}
y \\
z
\end{array}\right)\right\rangle_{H}=\int_{0}^{1}\left(y^{\prime}(x)+z(x)\right)^{2} d x+\int_{0}^{1} z^{\prime}(x)^{2} d x \geq 0
$$

and " $=0$ " if and only if $y=z \equiv 0$. Let $\left(\begin{array}{l}y_{1} \\ z_{1}\end{array}\right),\left(\begin{array}{l}y_{2} \\ z_{2}\end{array}\right) \in D(A)$ be given. Then it follows that

$$
\left\langle\left(\begin{array}{l}
y_{1} \\
z_{1}
\end{array}\right), A\left(\begin{array}{l}
y_{2} \\
z_{2}
\end{array}\right)\right\rangle_{H}=\left\langle A\left(\begin{array}{l}
y_{1} \\
z_{1}
\end{array}\right),\left(\begin{array}{l}
y_{2} \\
z_{2}
\end{array}\right)\right\rangle_{H} .
$$

Hence $A$ is positive and self-adjoint. This implies that $A$ has an orthonormal sequence of eigenelements $\left(\begin{array}{l}y_{j} \\ z_{j}\end{array}\right) \in D(A)(j \in \mathbb{N})$, and a corresponding sequence of eigenvalues $\lambda_{j} \in \mathbb{R}$ of finite multiplicity such that

$$
0<\lambda_{1} \leq \lambda_{2} \leq \ldots \leq \lambda_{j} \rightarrow \infty \quad \text { as } j \rightarrow \infty \text {. }
$$

The unique weak solution of (2.3) under the initial conditions (1.3) is then given by

$$
\left(\begin{array}{c}
w(x, t) \\
\xi(x, t)
\end{array}\right)=\sum_{j=1}^{\infty} \frac{1}{\sqrt{\lambda_{j}}} \int_{0}^{t} \sin \sqrt{\lambda_{j}}(t-s)\left\langle\left(\begin{array}{c}
f_{1}(\cdot, s) \\
f_{2}(\cdot, s)
\end{array}\right),\left(\begin{array}{l}
y_{j} \\
z_{j}
\end{array}\right)\right\rangle_{H} d s\left(\begin{array}{l}
y_{j} \\
z_{j}
\end{array}\right)
$$

for $x \in(0,1)$ and $t \geq 0$, and its time derivative reads

$$
\left(\begin{array}{c}
\dot{w}(x, t) \\
\dot{\xi}(x, t)
\end{array}\right)=\sum_{j=1}^{\infty} \int_{0}^{t} \cos \sqrt{\lambda_{j}}(t-s)\left\langle\left(\begin{array}{c}
f_{1}(\cdot, s) \\
f_{2}(\cdot, s)
\end{array}\right),\left(\begin{array}{c}
y_{j} \\
z_{j}
\end{array}\right)\right\rangle_{H} d s\left(\begin{array}{c}
y_{j} \\
z_{j}
\end{array}\right)
$$


Next we investigate the eigenvalue problem

$$
\left.\begin{array}{rl}
-y^{\prime \prime}(x)-z^{\prime}(x) & =\lambda y(x) \\
-z^{\prime \prime}(x)+y^{\prime}(x)+z(x) & =\lambda z(x)
\end{array}\right\} \quad(x \in(0,1))
$$

and

$$
\left.\begin{array}{r}
y(0)=z(0)=0 \\
y^{\prime}(1)+z(1)=0, z^{\prime}(1)=0
\end{array}\right\}
$$

Since we know according to (2.1) and (2.2) that the operator $A$ on $D(A)$ is positive and selfadjoint, this problem can only have a non-trivial solution, if $\lambda$ is real and positive. In addition we have

Lemma 2.1. The smallest eigenvalue $\lambda_{1}$ of the operator $A$ satisfies the estimate

$$
\lambda_{1}>1
$$

Proof. Let $\left(\begin{array}{l}y \\ z\end{array}\right) \in D(A)$. Then

$$
\left\langle\left(\begin{array}{l}
y \\
z
\end{array}\right), A\left(\begin{array}{l}
y \\
z
\end{array}\right)\right\rangle_{H}=\int_{0}^{1}\left(y^{\prime}(x)+z(x)\right)^{2} d x+\int_{0}^{1} z^{\prime}(x)^{2} d x
$$

Since $y(0)=z(0)=0$, the functions $y(x), z(x)$ satisfy Friedrichs' inequalities of the form

$$
\begin{gathered}
\int_{0}^{1} y^{2}(x) d x=\int_{0}^{1}\left(\int_{0}^{x} y^{\prime}(s) d s\right)^{2} d x \leq \int_{0}^{1} x \int_{0}^{x} y^{\prime}(s)^{2} d s d x \leq \frac{1}{2} \int_{0}^{1} y^{\prime}(x)^{2} d x \\
\int_{0}^{1} z^{2}(x) d x \leq \frac{1}{2} \int_{0}^{1} z^{\prime}(x)^{2} d x
\end{gathered}
$$

Taking this into account we deduce from $(*)$

$$
\begin{aligned}
\left\langle\left(\begin{array}{l}
y \\
z
\end{array}\right), A\left(\begin{array}{l}
y \\
z
\end{array}\right)\right\rangle_{H} \\
\quad=\int_{0}^{1}\left(\frac{1}{2} y^{\prime}(x)^{2}+z(x)^{2}\right) d x+\int_{0}^{1}\left(\frac{1}{2} y^{\prime}(x)^{2}+2 y^{\prime}(x) z(x)+z^{\prime}(x)^{2}\right) d x \\
\geq\left\langle\left(\begin{array}{l}
y \\
z
\end{array}\right),\left(\begin{array}{l}
y \\
z
\end{array}\right)\right\rangle_{H}+\frac{1}{2} \int_{0}^{1}\left(y^{\prime}(x)+2 z(x)\right)^{2} d x \\
\geq\left\langle\left(\begin{array}{l}
y \\
z
\end{array}\right),\left(\begin{array}{l}
y \\
z
\end{array}\right)\right\rangle_{H} .
\end{aligned}
$$


The latter relation proves that $\lambda_{1} \geq 1$. At the same time, assuming that

$$
A\left(\begin{array}{l}
y \\
z
\end{array}\right)=\left(\begin{array}{l}
y \\
z
\end{array}\right)
$$

we obtain $y^{\prime}(x)=-2 z(x)$. From here and from the equality $y^{\prime}(x)-z^{\prime \prime}(x)+z(x)=z(x)$ it follows that

$$
z^{\prime \prime}(x)+2 z(x)=0
$$

Since $z(0)=0, z^{\prime}(1)=0$ that leads to

$$
z(x) \equiv 0 .
$$

Then $y^{\prime}(x)=0, y(0)=0$ and, therefore, $y(x) \equiv 0$. Thus, $\lambda=1$ is not an eigenvalue of $A$. This completes the proof

We introduce functions

$$
\left.\begin{array}{l}
y_{1}(x)=y(x) \\
y_{2}(x)=y^{\prime}(x) \\
y_{3}(x)=z(x) \\
y_{4}(x)=z^{\prime}(x)
\end{array}\right\}
$$

and rewrite $(2.6)_{a}-(2.6)_{b}$ in the form

$$
\left(\begin{array}{l}
y_{1}^{\prime}(x) \\
y_{2}^{\prime}(x) \\
y_{3}^{\prime}(x) \\
y_{4}^{\prime}(x)
\end{array}\right)=\left(\begin{array}{cccc}
0 & 1 & 0 & 0 \\
-\lambda & 0 & 0 & -1 \\
0 & 0 & 0 & 1 \\
0 & 1 & 1-\lambda & 0
\end{array}\right)\left(\begin{array}{l}
y_{1}(x) \\
y_{2}(x) \\
y_{3}(x) \\
y_{4}(x)
\end{array}\right) \quad(x \in(0,1))
$$

and

$$
\left.\begin{array}{r}
y_{1}(0)=y_{3}(0)=0 \\
y_{2}(1)+y_{3}(1)=0 \\
y_{4}(1)=0
\end{array}\right\}
$$

Let us rewrite $(2.7)_{a}$ in the form

$$
y^{\prime}(x)=C y(x) \quad(x \in(0,1)) .
$$

In order to determine the general solution of this system we need the eigenvalues of $C$ which are given by

$$
\mu_{1}=\sqrt{-\lambda+\sqrt{\lambda}}, \quad \mu_{2}=-\mu_{1}, \quad \mu_{3}=\sqrt{-\lambda-\sqrt{\lambda}}, \quad \mu_{4}=-\mu_{3}
$$

and corresponding eigenvectors which are given by

$$
p_{1}=\left(\begin{array}{c}
1 \\
\mu_{1} \\
-\frac{\sqrt{\lambda}}{\mu_{1}} \\
-\sqrt{\lambda}
\end{array}\right), \quad p_{2}=\left(\begin{array}{c}
1 \\
-\mu_{1} \\
\frac{\sqrt{\lambda}}{\mu_{1}} \\
-\sqrt{\lambda}
\end{array}\right), \quad p_{3}=\left(\begin{array}{c}
1 \\
\mu_{3} \\
\frac{\sqrt{\lambda}}{\mu_{3}} \\
\sqrt{\lambda}
\end{array}\right), \quad p_{4}=\left(\begin{array}{c}
1 \\
-\mu_{3} \\
-\frac{\sqrt{\lambda}}{\mu_{3}} \\
\sqrt{\lambda}
\end{array}\right) .
$$


442

W. Krabs and G. M. Sklyar

The general solution of $(2.7)_{a}$ therefore reads

$$
y(x)=C_{1} e^{\mu_{1} x} p_{1}+C_{2} e^{-\mu_{1} x} p_{2}+C_{3} e^{\mu_{3} x} p_{3}+C_{4} e^{-\mu_{3} x} p_{4} \quad(x \in[0,1])
$$

where $C_{1}, C_{2}, C_{3}, C_{4} \in \mathbb{C}$ are variable constants. The boundary conditions $(2.7)_{b}$ lead to the conditions

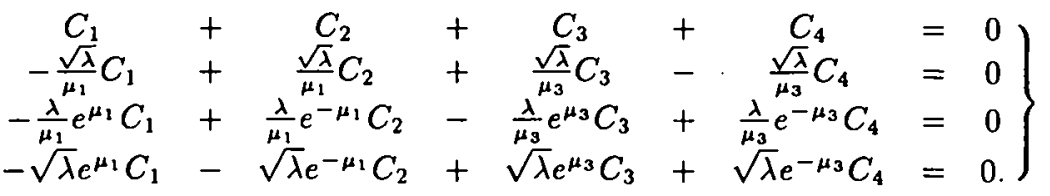

A necessary and sufficient condition for this system to have a nontrivial solution is that

$$
\operatorname{det}\left(\begin{array}{cccc}
1 & 1 & 1 & 1 \\
-\frac{\sqrt{\lambda}}{\mu_{1}} & \frac{\sqrt{\lambda}}{\mu_{1}} & \frac{\sqrt{\lambda}}{\mu_{3}} & \frac{\lambda}{\mu_{3}} \\
-\frac{\lambda}{\mu_{1}} e^{\mu_{1}} & \frac{\lambda}{\mu_{1}} e^{-\mu_{1}} & -\frac{\lambda}{\mu_{3}} e^{\mu_{3}} & \frac{\lambda}{\mu_{3}} e^{-\mu_{3}} \\
-\sqrt{\lambda} e^{\mu_{1}} & -\sqrt{\lambda} e^{-\mu_{1}} & \sqrt{\lambda} e^{\mu_{3}} & \sqrt{\lambda} e^{-\mu_{3}}
\end{array}\right)=0 .
$$

This is equivalent to

$$
8 \mu_{1} \mu_{3}+\left(\mu_{1}+\mu_{3}\right)^{2}\left(e^{\mu_{1}+\mu_{3}}+e^{-\mu_{1}-\mu_{3}}\right)-\left(\mu_{1}-\mu_{3}\right)^{2}\left(e^{\mu_{1}-\mu_{3}}+e^{-\mu_{1}+\mu_{3}}\right)=0 .
$$

By Lemma 2.1 we know that $\lambda>1$, hence $\lambda>\sqrt{\lambda}$ and

$$
\mu_{1}=i \sigma_{1}, \quad \sigma_{1}=\sqrt{\lambda-\sqrt{\lambda}}, \quad \mu_{3}=i \sigma_{3}, \quad \sigma_{3}=\sqrt{\lambda+\sqrt{\lambda}}
$$

If we put $u=\sigma_{3}-\sigma_{1}$ and $v=\sigma_{3}+\sigma_{1}$, then (2.8) turns out to be equivalent to

$$
u^{2}(1+\cos u)=v^{2}(1+\cos v) \text {. }
$$

On defining $\omega=\sqrt{\lambda}(>1)$ we obtain $v=\sqrt{\omega^{2}+\omega}+\sqrt{\omega^{2}-\omega}$, hence $v^{2}=2 \omega^{2}+$ $2 \sqrt{\omega^{4}-\omega^{2}}$ which implies $v^{4}=4 \omega^{2}\left(v^{2}-1\right)$. This leads to

$$
\omega=\frac{v^{2}}{2 \sqrt{v^{2}-1}} \text {. }
$$

Since $\lambda>1$, it follows that $v>\sqrt{2}$, so that $(2.10)$ defines a bijection between $(\sqrt{2}, \infty)$ and $(1, \infty)$ which allows to find $\omega \in(1, \infty)$ for every $v \in(\sqrt{2}, \infty)$. We also note that $\omega \sim \frac{v}{2}$ when $v \rightarrow \infty$.

Further, we have $u=\sqrt{\omega^{2}+\omega}-\sqrt{\omega^{2}-\omega}$, hence $u^{2}=2\left(\omega^{2}-\sqrt{\omega^{4}-\omega^{2}}\right)$. Substituring $\omega$ from (2.10) we obtain

$$
\begin{aligned}
u^{2} & =2\left(\frac{v^{4}}{4\left(v^{2}-1\right)}-\sqrt{\frac{v^{8}}{16\left(v^{2}-1\right)^{2}}-\frac{v^{4}}{4\left(v^{2}-1\right.}}\right) \\
& =\frac{1}{2}\left(\frac{v^{4}}{v^{2}-1}-\frac{v^{2}\left(v^{2}-2\right)}{v^{2}-1}\right) \\
& =\frac{v^{2}}{v^{2}-1} .
\end{aligned}
$$


Therefore

$$
u=u(v)=\frac{v}{\sqrt{v^{2}-1}} \rightarrow 1 \quad \text { as } \quad v \rightarrow \infty
$$

Substituting $u=u(v)$ into the spectral equation (2.9) we obtain

$$
1+\cos \left(\frac{v}{\sqrt{v^{2}-1}}\right)=\left(v^{2}-1\right)(1+\cos v) \text {. }
$$

Conclusion. Each eigenvalue of $(2.6)_{a}-(2.6)_{b}$ can be found as

$$
\lambda=\frac{v^{4}}{4\left(v^{2}-1\right)}
$$

where $v>\sqrt{2}$ is a solution of (2.11).

Next we show the following two statements:

(1) For every $k \geq 2$ there exist two solutions $v_{1}^{(k)} \in[2(k-1) \pi,(2 k-1) \pi)$ and $v_{2}^{(k)} \in$ $((2 k-1) \pi, 2 k \pi]$ of $(2.11)$.

(2) $\lim _{k \rightarrow \infty}\left[(2 k-1) \pi-v_{1}^{(k)}\right]=\lim _{k \rightarrow \infty}\left[v_{2}^{(k)}-(2 k-1) \pi\right]=0$.

For the proof we rewrite (2.11) in the form

$$
v=\varphi(v)=\sqrt{1+\frac{1+\cos \frac{v}{\sqrt{v^{2}-1}}}{1+\cos v}} .
$$

In order to prove (1) we notice the following:

(a) $\varphi(2 k \pi) \leq \sqrt{1+\frac{1+1}{1+\cos 2 k \pi}}=\sqrt{2} \leq \pi$ for $k \in \mathbb{N}$.

(b) The function $\varphi(v)$ is continuous on the intervals $[(2 k-2) \pi,(2 k-1) \pi)$ and $((2 k-1) \pi, 2 k \pi](k \geq 2)$.

(c) $\varphi(v) \rightarrow \infty$ as $v \rightarrow(2 k-1) \pi \quad(k \in \mathbb{N})$.

Hence (2.12) or equivalently (2.11) has at least one solution on each interval [2(k$1) \pi,(2 k-1) \pi)$ and $((2 k-1) \pi, 2 k \pi]$ for $k \in \mathbb{N}$.

In order to prove (2) we notice that the inequality

$$
\varphi(v) \leq \sqrt{1+\frac{2}{1+\cos v}}<2(k-1) \pi \leq v
$$

holds true when

$$
v \in\left[2(k-1) \pi, \arccos \left(\frac{2}{(2(k-1) \pi)^{2}-1}-1\right)+(2 k-2) \pi\right)
$$

and

$$
v \in\left(-\arccos \left(\frac{2}{(2(k-1) \pi)^{2}-1}-1\right)+2 k \pi, 2 k \pi\right]
$$


for $k \geq 2$. So the roots $v_{1,2}^{(k)}$ belong to the interval

$$
\Delta_{k}=\left[\arccos \left(\frac{2}{(2(k-1) \pi)^{2}-1}\right)+(2 k-2) \pi, 2 k \pi-\arccos \left(\frac{2}{2(k-1) \pi)^{2}-1}-1\right)\right]
$$

for $k \geq 2$. Since

$$
\arccos \left(\frac{2}{(2(k-1) \pi)^{2}-1}-1\right) \rightarrow \pi \quad \text { if } k \rightarrow \infty
$$

the length of $\Delta_{k}$ tends to 0 when $k \rightarrow \infty$. This completes the proof of (2).

One can also prove the uniqueness of the solutions $v_{1}^{(k)}$ and $v_{2}^{k)}$ of (2.12) or equivalently $(2.11)$ in $[2(k-1) \pi,(2 k-1) \pi)$ and $((2 k-1) \pi, 2 k \pi]$, respectively, as a result of more complicated arguments. Summarizing we obtain

Lemma 2.2. The eigenvalues $\lambda_{k}$ of $(2.6)_{a}-(2.6)_{b}$ for large $k$ are of the form

$$
\lambda_{n}= \begin{cases}\frac{1}{4}\left[(2 k-1) \pi-\varepsilon_{2 k-1}\right]^{2} & \text { if } n=2 k-1 \\ \frac{1}{4}\left[(2 k-1) \pi+\varepsilon_{2 k}\right]^{2} & \text { if } n=2 k\end{cases}
$$

where $0<\varepsilon_{2 k-1}, \varepsilon_{2 k}$ and $\lim _{n \rightarrow \infty} \varepsilon_{n}=0$.

\section{On the solution of the problem of controllability}

From (2.4) and (2.5) it follows that the end conditions (1.5) are equivalent to

$$
\begin{aligned}
& a_{j} \int_{0}^{T} \sin \sqrt{\lambda_{j}}(T-t) \ddot{\theta}(t) d t=0 \\
& \text { and } \quad(j \in \mathbb{N}) \\
& a_{j} \int_{0}^{T} \cos \sqrt{\lambda_{j}}(T-t) \ddot{\theta}(t) d t=0
\end{aligned}
$$

where

$$
a_{j}=-\int_{0}^{1}(r+x) y_{j}(x) d x+\int_{0}^{1} z_{j}(x) d x
$$

The end conditions (1.4) are equivalent to

$$
\int_{0}^{T} \ddot{\theta}(t) d t=0 \quad \text { and } \quad \int_{0}^{T} t \ddot{\theta}(t) d t=-\theta_{T}
$$

Now let us consider the following 
Moment Problem. Find $u \in L^{2}(0, T)$ such that

$$
\left.\begin{array}{rl}
\int_{0}^{T} u(t) d t & =0 \\
\int_{0}^{T} t u(t) d t & =-\theta_{T} \\
\int_{0}^{T} \cos \sqrt{\lambda_{j}} t u(t) d t & =0 \\
\int_{0}^{T} \sin \sqrt{\lambda_{j}} t u(t) d t & =0 .
\end{array}\right\}
$$

If $u \in L^{2}(0, T)$ is a solution of (3.4), then $\ddot{\theta}(t)=u(T-t)(t \in[0, T])$ solves $(3.1),(3.3)$ and $\theta(t)=\int_{0}^{t}(t-s) u(T-s) d s \quad(t \in[0, T])$ solves the problem of controllability. Therefore, in order to find a solution of the problem of controllability we have to find a solution $u \in L^{2}(0, T)$ of the moment problem (3.4).

We shall show later that, if $T>4$, the function $f(t)=t(t \in[0, T])$ does not belong to the closure of the span of

$$
\left\{1, \cos \sqrt{\lambda_{j}} t, \sin \sqrt{\lambda_{j}} t \mid t \in[0, T], j \in \mathbb{N}\right\}
$$

which we denote by $W$. This implies that there is exactly one function $\hat{w} \in W$ such that

$$
0<\|\hat{w}-f\|_{L^{2}(0, T)} \leq\|w-f\|_{L^{2}(0, T)} \quad \text { for all } w \in W
$$

which is characterized by

$$
\int_{0}^{T}(f(t)-\hat{w}(t)) w(t) d t=0 \quad(w \in W) .
$$

This implies

$$
\left.\begin{array}{r}
\int_{0}^{T}(f(t)-\hat{w}(t)) d t=0 \\
\int_{0}^{T} \cos \sqrt{\lambda_{j}} t(f(t)-\hat{w}(t)) d t=0 \\
\int_{0}^{T} \sin \sqrt{\lambda_{j}} t(f(t)-\hat{w}(t)) d t=0
\end{array}\right\} \quad(j \in \mathbb{N})
$$


and

$$
\int_{0}^{T}(f(t)-\hat{w}(t)) f(t) d t=\|\hat{w}-f\|_{L^{2}(0, T)}^{2}>0 .
$$

If we define

$$
u(t)=-\frac{\theta_{T}}{\|\hat{w}-f\|_{L^{2}(0, T)}^{2}}(f(t)-\hat{w}(t)) \quad(t \in[0, T]),
$$

then $u \in L^{2}(0, T)$ and solves the moment problem (3.4). Thus the problem of controllability is solvable, if $T>4$.

In order to show that $f \notin W$, if $T>4$, we make use of [3: Theorem 1.2.17] which reads as follows:

If

$$
\limsup _{x \rightarrow \infty} \limsup _{y \rightarrow \infty} \frac{d(x+y)-d(x)}{y}<\frac{T}{2 \pi}
$$

where, for every $x>0, d(x)=\max \left\{k \in \mathbb{N} \mid \sqrt{\lambda_{k}}<x\right\}$, then the system

$$
\left\{1, t, \sin \sqrt{\lambda_{k}} t, \cos \sqrt{\lambda_{k}} t \mid t \in[0, T], k \in \mathbb{N}\right\}
$$

is minimal in $L^{2}(0, T)$ which implies that $f \notin W$.

Let $x>1$ be large. Then $\sqrt{\lambda_{n}}<x$ implies $n<\frac{2 x}{\pi}+\frac{\alpha}{\pi}+1$ where $\alpha>0$ is a constant with $\left|\varepsilon_{k}\right| \leq \alpha$ for all $k \in \mathbb{N}$. This implies

$$
d(x)<\frac{2 x}{\pi}+\frac{\alpha}{\pi}+1 \quad \text { hence } \quad d(x+y)<\frac{2(x+y)}{\pi}+\frac{\alpha}{\pi}+1
$$

for all $x>1$ and $y>0$.

Let again $x>1$ be given sufficiently large. Then we put $n=\left[\frac{2 x}{\pi}-\frac{\alpha}{\pi}\right]\left(\leq \frac{2 x}{\pi}-\frac{\alpha}{\pi}\right)$ and conclude $\sqrt{\lambda_{n}}<\frac{1}{2}(n \pi+\alpha) \leq x$, hence $\frac{2 x}{\pi}-\frac{\alpha}{\pi}-1 \leq d(x)$. For every $y>0$ we then obtain

$$
\frac{d(x+y)-d(x)}{y}<\frac{2}{\pi}+\frac{2 \alpha}{y \pi}+\frac{2}{y} \text {. }
$$

This implies

$$
\limsup _{x \rightarrow \infty} \limsup _{y \rightarrow \infty} \frac{d(x+y)-d(x)}{y} \leq \frac{2}{\pi}<\frac{T}{2 \pi}
$$

if $T>4$. Summarizing we have the

Theorem 3.1. If $T>4$, then the problem of controllability is solvable. 


\section{Concluding remarks}

In a forthcoming paper it will be shown that all the $a_{j}$ 's defined by (3.2) and appearing in (3.1) are unequal to zero unless the radius of the disk is of the form

$$
r=\frac{\sigma_{1} \sin \sigma_{1}-\sigma_{3} \sin \sigma_{3}}{\sqrt{\lambda}\left(\cos \sigma_{3}-\cos \sigma_{1}\right)}
$$

with $2 \pi k<\sigma_{3}+\sigma_{1}<\pi+2 \pi k$, in which case $r$ is called singular.

If $r$ is singular, then $a_{j}=0$ for at least one $j \in \mathbb{N}$ and for initial states which are not positions of rest even approximate controllability to a state of rest is impossible.

If $r$ is non-singular, then approximate controllability from an initial state of finite energy to the states of rest is guaranteed for $T>4$. This is also a consequence of the minimality of the system $\left\{1, t, \sin \sqrt{\lambda_{k}} t, \cos \sqrt{\lambda_{k}} t, \mid t \in[0, T], k \in \mathbb{N}\right\}$ in $L^{2}(0, T)$ for $T>4$.

\section{References}

[1] Ballas, K.: Steuerung eines rotierenden, flexiblen Balkens mit einem Drehmoment von minimaler $L^{2}$-Norm. Diplomarbeit. Darmstadt: Techn. Univ. 1994.

[2] Delfour, M. C., Kern, M., Passeron, L. and B. Sevenne: Modelling of a rotating flexible beam. In: Control of Distributed Parameter Systems (ed.: H. E. Rauch). Los Angeles: Pergamon Press 1986, pp. 383 - 387.

[3] Krabs, W.: On Moment Theory and Controllability of One-Dimensional Vibrating Systems and Heating Processes (Lecture Notes in Control and Information Sciences: Vol. 173). Berlin et al.: Springer-Verlag 1992.

[4] Krabs, W.: Controllability of a rotating beam. In: Analysis and Optimization of Systems: State and Frequency Domain Approaches for Infinite-Dimensional Systems (Lecture Notes in Control and Information Sciences: Vol. 185; ed.: R. F. Curtain). Berlin: SpringerVerlag 1993, pp. 447 - 458.

[5] Krabs, W.: On the controllability of the rotation of a flexible arm. In: Control and Estimation of Distributed Parameter Systems: Nonlinear Phenomena (Int. Ser. Num. Math.: Vol. 118; ed.: W. Desch et al.). Basel: Birkhäuser Verlag 1994, pp. $267-269$.

[6] Krabs, W. and Chi-Long Nguyen: On the controllability of a robot arm. Math. Meth. Appl. Sci. 21 (1998), $25-42$.

[7] Leugering, G.: Control and stabilization of a flexible arm. Dynamics and Stability of Systems 5 (1990), 37 - 46.

[8] Leugering, G.: On control and stabilization of a rotating beam by applying moments at the base only. In: Optimal Control of Partial Differential Equations (Lecture Notes in Control and Information Sciences: Vol. 149; eds.: K. H. Hoffmann and W. Krabs). Berlin: Springer-Verlag 1991, $182-191$.

[9] Sakawa, Y., Ito, R. and N. Fujii: Optimal control of a flezible arm. In: Control Theory for Distributed Parameter Systems and Applications (Lecture Notes in Control and Information Sciences: Vol. 54; eds.: K. Kappel et al.). Berlin: Springer-Verlag 1983, pp. $175-187$. 
[10] Xiong, Xiao-Jin: Modelling, Control and Computer Simulation of a Rotating Timoshenko Beam. Ph.D. Thesis. Montréal: McGill Univ. 1997.

Received 12.05.1998 